\title{
High Quality Ti-Implanted Si Layers Above Solid Solubility Limit
}

\author{
J. Olea, D. Pastor, M. Toledano-Luque, E. San-Andrés, I. Mártil, and G. González-Díaz \\ Dpto. de Física Aplicada III (Electricidad y Electrónica). Facultad de Ciencias Físicas. Universidad \\ Complutense de Madrid. E-28040 Madrid. Spain
}

\begin{abstract}
In this work we report the successful doping of Si with $\mathrm{Ti}$ at doses beyond the Mott limit for this element keeping high lattice quality. Ti implantation in $\mathrm{Si}$ at high doses and subsequent Pulsed-Laser Melting (PLM) annealing have been performed. Time-of-Flight Secondary Ion Mass Spectroscopy (SIMS) measurements confirm that Ti concentration exceed the Mott limit in the implanted layer, and Glancing Incidence X-Ray Diffraction (GIXRD) and Transmission Electron Microscopy (TEM) measurements prove that good crystallinity can be achieved over solid solubility limit. Hall effect characterization points out a high electrical activation and high mobility in all samples.
\end{abstract}

\section{INTRODUCTION}

In the last decade, an enormous effort has been channeled towards the improvement of the efficiency in the solar cell technology [1]. One of the most promising concepts is the intermediate band solar cell (IBSC) [2], which has been leading the so-called Third Generation of photovoltaic technology [3]. The IBSC is a forward-looking approach that can overcome the theoretical solar conversion efficiency limit for single junction solar cells [4]. This new technology is based on the idea of placing a new band of allowed states between the traditional valence and conduction bands that permits photons of energy lower than the semiconductor bandgap to be absorbed, yielding a higher efficiency. Apart from the optical transition from the valence band to the

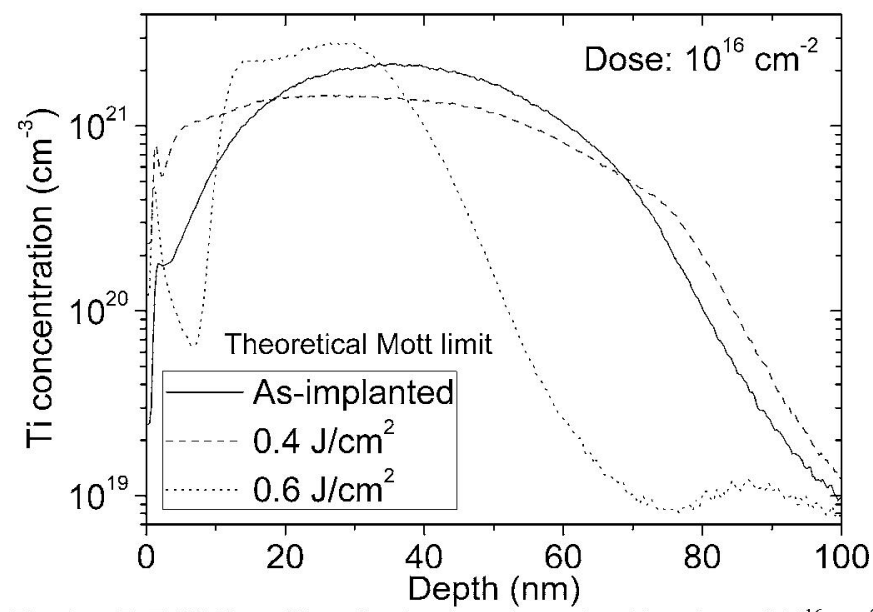

Fig. 1. ToF-SIMS profiles of an implanted sample with a dose of $10^{16} \mathrm{~cm}^{-2}$ and two samples implanted with the same dose and PLM annealed at energy densities of 0.4 and $0.6 \mathrm{~J} / \mathrm{cm}^{2}$. conduction band, two new transitions could show up: one from the valence band to the intermediate band (IB) and another from the IB to the conduction band. Then, in an IB material an electron can be pumped from the valence band to the conduction band by one stage transition or by two stages transition. This capacity permits to waste less incident photons, which translates in a higher efficiency conversion.

Among other possibilities like quantum dots [5] or highlymismatched alloys [6], ion implantation of deep level impurities can fulfill this task if the concentration is high enough to exceed the Mott limit [7]. Usually this involves breaking the solubility limit [8], which can be reached by using highly non-equilibrium techniques, i.e., ion implantation and subsequent Pulsed-Laser Melting (PLM) annealing. In fact, PLM technique has been extensively used to design intermediate band (IB) materials [9]. Furthermore, theoretical [10] and experimental [11] results show that $\mathrm{Ti}$ is a promising deep level candidate.

Electrical and structural characterization of IB materials designed by ion implantation of deep level impurities are mandatory due to the high concentration needed to reach the Mott limit. In this paper we demonstrate that $\mathrm{Ti}$ in concentrations over the Mott limit in silicon can be introduced keeping high lattice quality and showing significant electrical activation.

\section{EXPERIMENTAL}

$300 \mu \mathrm{m}$ (111) n-type silicon samples $\left(\mu=1500 \mathrm{~cm}^{2} / \mathrm{Vs} ; \mathrm{n}=\right.$ $2.2 \times 10^{13} \mathrm{~cm}^{-3}$ at room temperature) were implanted in a VARIAN CF3000 Ion Implanter at $30 \mathrm{KeV}$ with Ti at high doses $\left(10^{15}, 5 \times 10^{15}, 10^{16}\right.$ and $\left.5 \times 10^{16} \mathrm{~cm}^{-2}\right)$. After implantation samples were PLM annealed with a $20 \mathrm{~ns} \mathrm{KrF}$ excimer laser $(248 \mathrm{~nm})$ at $0.2,0.4,0.6$ and $0.8 \mathrm{~J} / \mathrm{cm}^{2}$ at J.P. Sercel Associates Inc. (New Hampshire, USA).

The structural characterization of the samples was carried out by X-ray diffraction, Transmission Electron Microscopy (TEM) and Time-of-Flight Secondary Ion Mass Spectroscopy (ToF-SIMS). Glancing-Incidence X-Ray Diffraction (GIXRD) patterns were obtained by a Panalytical X'Pert PRO MRD diffractometer working with a $\mathrm{Cu} \mathrm{K}_{\alpha}$ source. The GIXRD patterns were collected at different incidence angles from 0.1 to $3^{\circ}$ to study the crystal structure of the samples at different depths. Cross sectional TEM images were obtained by a JEOL JEM-2000FX working at $200 \mathrm{keV}$. Simultaneously with the 
Proceedings of the 2009 Spanish Conference on Electron Devices - Feb 11-13, 2009. Santiago de Compostela, Spain.

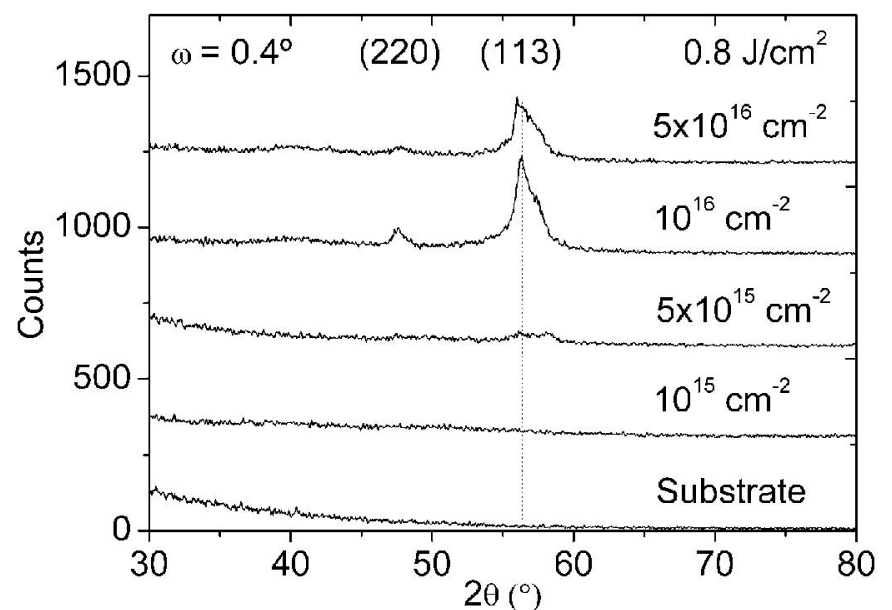

Fig. 2. GIXRD diffractograms at a incidence angle of $0.4^{\circ}$ for the samples implanted with different doses and annealed at $0.8 \mathrm{~J} / \mathrm{cm}^{2}$.

TEM measurements, Electron Diffraction (ED) patterns with a selected area of diffraction of about $50 \mathrm{~nm}$ were obtained. The ED images provided information on the crystalline morphology of the implanted layer. The microscope is also equipped with an Energy Dispersive X-ray (EDX) spectrometer, which provides local information of the chemical composition of the sample. High Resolution TEM (HRTEM) images were obtained by a JEM-3000F working at $300 \mathrm{keV}$. ToF-SIMS measurements were performed with a TOF_SIMS IV model manufactured by ION-TOF, using a 25 $\mathrm{KeV}$ pulsed $\mathrm{Ga}^{+}$ion beam at $45^{\circ}$ incidence and $\mathrm{O}_{2}$ flow.

Hall effect measurements at room temperature were carried out by the van der Pauw set up with a Keithley SCS 4200 model, feeding the electromagnet with a bipolar Kepco BOP 50-20MG power supply. Changing the polarity of the measurement current source and the direction of the magnetic flux the thermo-galvanomagnetic effects are reduced.

\section{RESULTS}

Fig. 1 shows the Ti depth profile for the as-implanted sample with $10^{16} \mathrm{~cm}^{-2}$ and two samples implanted with the same dose and PLM annealed. The sample annealed at 0.4 $\mathrm{J} / \mathrm{cm}^{2}$ has the typical diffused profile, flattened with respect to the as-implanted. On the contrary, the profile of the sample annealed at $0.6 \mathrm{~J} / \mathrm{cm}^{2}$ has been pushed to the surface, becoming steeper and with a higher maximum. The valley at $75 \mathrm{~nm}$ in the profile of the $0.6 \mathrm{~J} / \mathrm{cm}^{2}$ annealed sample could indicate the depth of the melted region. The area beyond this point would be only heated but not melted. This figure points out that an energy density under $0.4 \mathrm{~J} / \mathrm{cm}^{2}$ is not capable of melting the surface, while higher energy densities melt the surface and push the impurities by solid-liquid solubility difference interaction, causing a snow-plow effect and recrystallization. Melting of at least up to the implanted depth is a must in order to anneal in non-equilibrium regime and surpass the solid solubility limit, and this occurs when the energy density is equal to or higher than $0.6 \mathrm{~J} / \mathrm{cm}^{2}[12]$.

Fig. 2 shows the $x$-ray diffractograms at $0.4^{\circ}$ glancing angle for the samples PLM annealed at $0.8 \mathrm{~J} / \mathrm{cm}^{2}$ and implanted with different doses. For the samples implanted with the highest

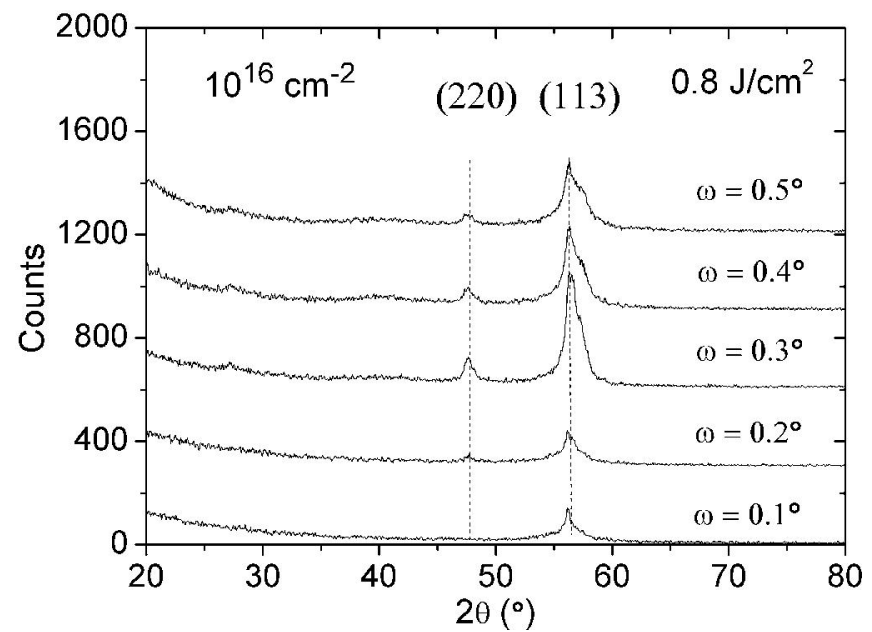

Fig. 3. GLXRD diffractograms at different incidence angles for the sample implanted with a $10^{16} \mathrm{~cm}^{-2}$ dose and annealed at $0.8 \mathrm{~J} / \mathrm{cm}^{2}$.

doses $\left(5 \times 10^{16}\right.$ and $\left.10^{16} \mathrm{~cm}^{-2}\right)$, two diffraction peaks at $47.1^{\circ}$ and $55.9^{\circ}$ can be observed. For the sample with a dose of $5 \times 10^{15} \mathrm{~cm}^{-2}$, the peak placed at $47.1^{\circ}$ disappears, but the peak at $55.9^{\circ}$ is still present. However, both diffraction peaks vanish for the sample implanted with the lowest dose $\left(10^{15} \mathrm{~cm}^{-2}\right)$. These peaks are attributed to the (220) and (113) silicon reflections. It is worth highlighting that no peaks associated to Ti-Si phases are present. These results point out the formation of a silicon polycrystalline structure for the samples implanted with the highest doses. To further study the crystal structure at different depths, the x-ray diffractograms were collected at different glancing angles.

Fig. 3 shows the GIXRD at different glancing angles for the sample implanted with a $10^{16} \mathrm{~cm}^{-2}$ and annealed at $0.8 \mathrm{~J} / \mathrm{cm}^{2}$. The intensities of the (220) and (113) silicon reflections increase with increasing the glancing incidence angle up to $0.3^{\circ}$, and decreases with higher incidence angles. Analogous behavior was observed for the other samples. This trend hints at the formation of a silicon polycrystalline phase in the implanted layer for the samples implanted with the highest doses. However, no diffraction peaks were observed at any incidence angle for the sample implanted with the lowest dose $\left(10^{15} \mathrm{~cm}^{-2}\right)$, pointing to a good crystallization of the implanted layer. From these results it is concluded that good recrystallization is only obtained at the lower implanted doses $\left(10^{15} \mathrm{~cm}^{-2}\right)$ and annealed at the highest energy density $(0.8$ $\mathrm{J} / \mathrm{cm}^{2}$ ).

Further confirmation of crystalline structure of the different samples comes from the TEM images and ED measurements. Fig. 4 shows the cross sectional TEM images of the sample implanted with different doses, and annealed at $0.8 \mathrm{~J} / \mathrm{cm}^{2}$. In the images (a) and (b) it can be observed a polycrystalline layer on top of a monocrystalline silicon substrate. EDX measurements confirmed that the polycrystalline layer contained silicon and titanium. The thicknesses of these polycrystalline layers were about $50 \mathrm{~nm}$ supporting ToF-SIMS measurements. In the images (a) and (b) it is also appreciable the increase in grain size with decreasing the implanted dose. The sample implanted with the highest dose presents a nanocrystalline structure, with a mean grain size of $5 \mathrm{~nm}$. 


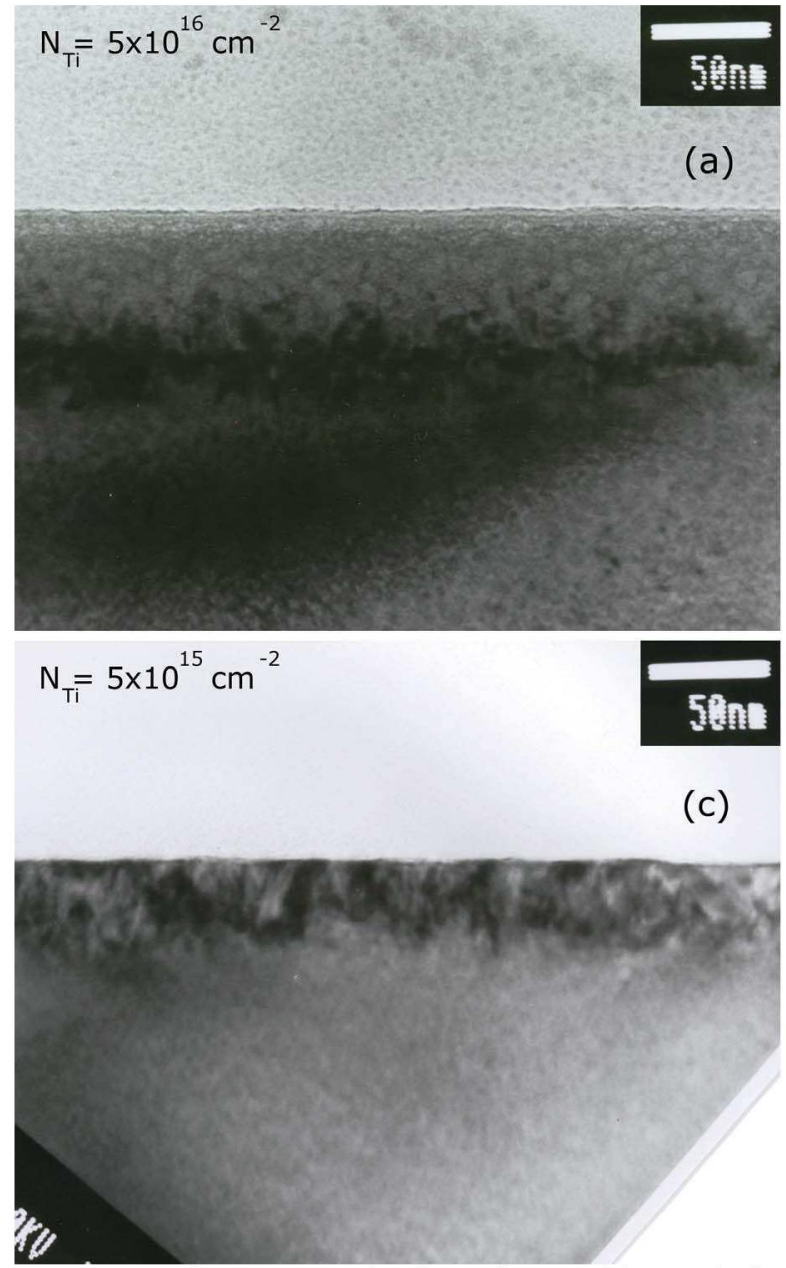

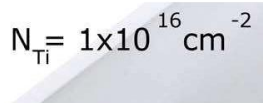

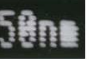

(b)

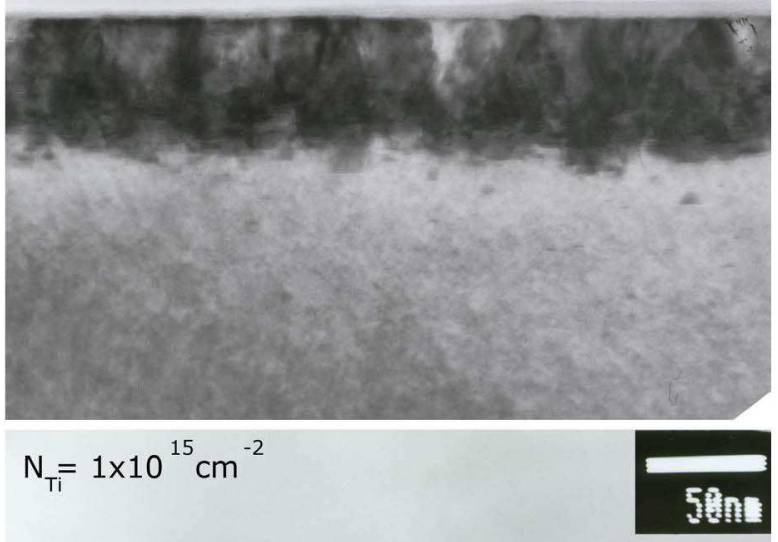

(d)

Fig. 4. Cross sectional TEM images of the samples implanted with different doses, and annealed at $0.8 \mathrm{~J} / \mathrm{cm}^{2}$.

When decreasing the implanted dose, the grain size presents a dimension comparable to the implanted layer thickness.

Fig. 5 shows the electron diffraction pattern for these two samples. From the first image it can be confirmed that, as was observed by TEM, the sample implanted with the highest dose is polycrystalline with small grains. This is indicated by the diffraction dots which are grouped in concentric ring around the direct beam. On the other, the electron diffraction image of the sample implanted with a $10^{16} \mathrm{~cm}^{-2}$ dose presented an unusual pattern: the bright spots correspond to the [111] zone axis of the silicon, but intermediated spots appear between the
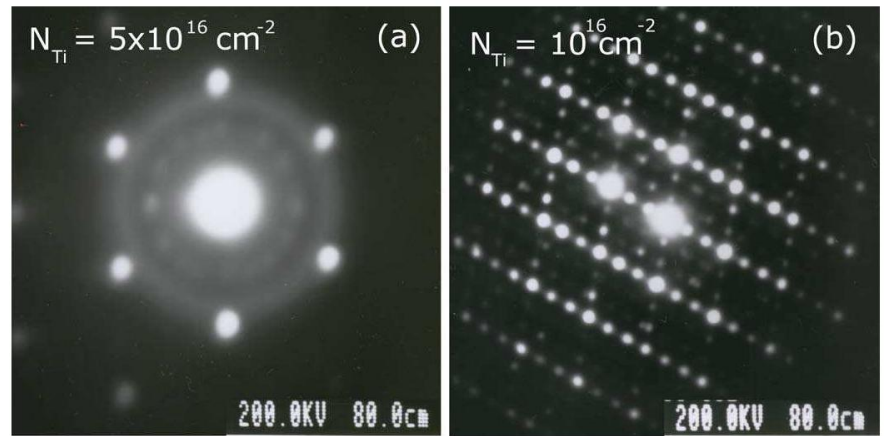

Fig. 5. Electron diffraction images of the implanted layer for the samples annealed at $0.8 \mathrm{~J} / \mathrm{cm}^{2}$ and implanted with a dose of $5 \times 10^{16} \mathrm{~cm}^{-2}$ (left), and $10^{16}$ $\mathrm{cm}^{-2}$ (right). main dots. This effect is due to the highly defective lattice reconstruction [13].

Fig. 4 (c) and (d) shows the TEM images for the samples with the lower doses. For the sample implanted with a dose of $5 \times 10^{15} \mathrm{~cm}^{-2}$, the thickness of the defective implanted layer is reduced down to about $30 \mathrm{~nm}$. For the sample with a $10^{15} \mathrm{~cm}^{-2}$ dose, no contrast difference appears between the implanted layer, thus, the defective layer is suppressed. In addition, the electron diffraction patterns of the implanted layer and the silicon substrate did not show difference. This result points to a good reconstruction of the lattice, and strengthens the GIXRD results.

Finally, High-Resolution TEM was used to further study this sample. Fig. 6 shows the HRTEM images of the titanium implanted sample and of unimplanted silicon substrate. Areas with a different contrast were observed seldom-distributed in the implanted layer. This effect is likely due to stacking faults.

Effective Hall mobility and effective sheet carrier concentration of the implanted-layer/substrate bilayer shown in Fig. 7 demonstrate that Ti-doped Si has been achieved. The high mobility measured confirms the good quality of the implanted layer after PLM process even though the dose was very high. For the sample implanted with the lowest dose the mobility is almost equal to the substrate mobility. Carrier concentration increases with higher doses as opposite to mobility following the usual phonon scattering trend. These 

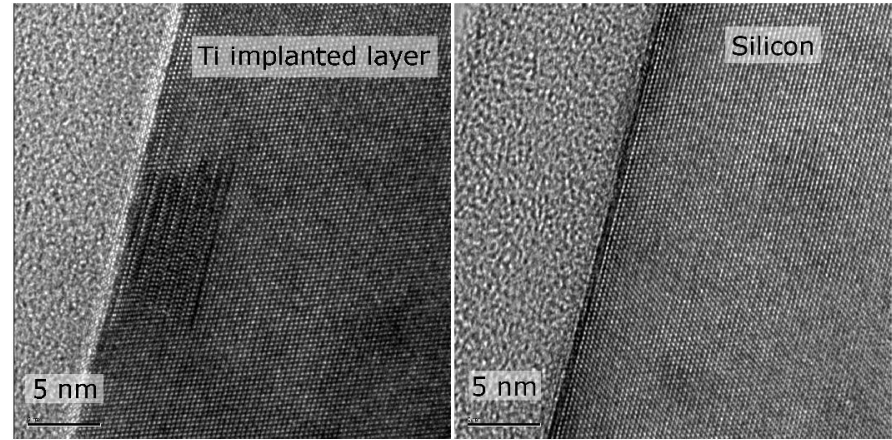

Fig. 6. High Resolution TEM images of the sample implanted with a dose of $10^{15} \mathrm{~cm}^{-2}$ and PLM annealed at $0.8 \mathrm{~J} / \mathrm{cm}^{2}$, and of unimplanted substrate.

parameters are an effective measurement related to both the implanted layer, which has a depth of a few tens of nanometers, and the substrate $(300 \mu \mathrm{m})$. As the substrate thickness is much higher than the layer thickness (more than three orders of magnitude), the actual carrier concentration in the implanted layer must be several orders of magnitude higher than the effective one, otherwise it could not affect the effective value measured. This result indicates that the implanted layer sheet carrier concentration and substrate sheet carrier concentration have to be at least comparable, concluding that we have achieved an actual high electrical activation of $\mathrm{Ti}$ in silicon. Moreover, the effective carrier concentration of the sample implanted with $10^{16} \mathrm{~cm}^{-2}$ is already higher than the solid solubility limit of $\mathrm{Ti}$ in $\mathrm{Si}\left(2 \times 10^{14}\right.$ $\left.\mathrm{cm}^{-3}\right)[8]$.

\section{CONCLUSIONS}

From the GIXRD results and TEM images, it can be concluded that the implanted layer with the lowest dose and annealed with the highest laser energy density presents a suitable reconstruction of the crystal structure, taking into account that the lowest dose $\left(10^{15} \mathrm{~cm}-2\right)$ is actually high. This result is supported by the high mobility measured for all the samples, which for the lowest dose implanted is equal to the substrate mobility. To recrystallize the damaged layer the energy density of the PLM annealing must be at least 0.6 $\mathrm{J} / \mathrm{cm}^{2}$. A very high activation of Ti impurities is achieved, and solid solubility limit of $\mathrm{Ti}$ in $\mathrm{Si}$ is surpassed with high mobility. This is a remarkable result, and Ti-implanted Si could be a prototype for IB materials based on deep level impurities.

\section{ACKNOWLEDGMENT}

Authors would like to acknowledge the Nanotechnology and Surface Analysis Services of the Universidad de Vigo C.A.C.T.I. for SIMS measurements, C.A.I. de Difracción de Rayos X of the Universidad Complutense de Madrid for GIXRD measurements, C.A.I. de Técnicas Físicas of the Universidad Complutense de Madrid for ion implantation experiments and C.A.I. de Microscopía Electronica "Luis Bru" for TEM images

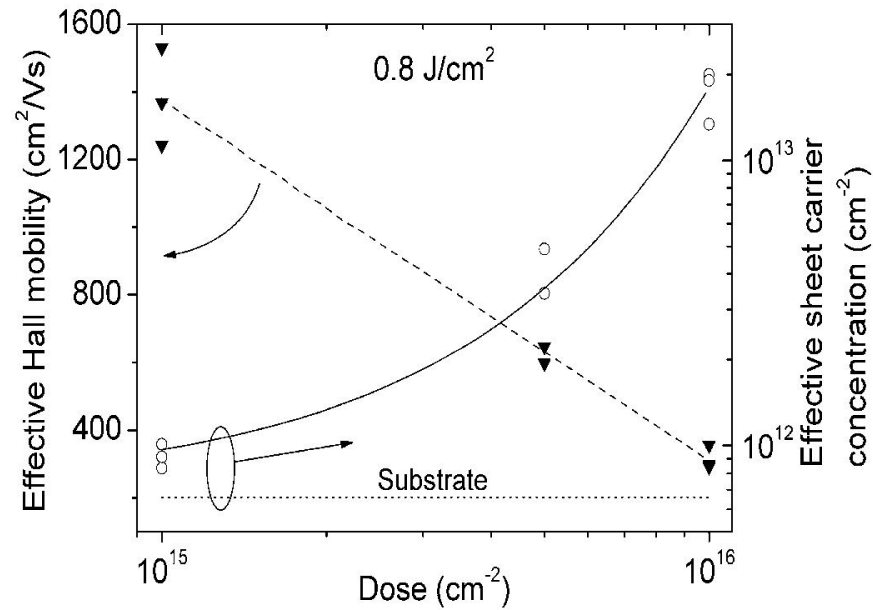

Fig. 7. Effective Hall mobility $(\boldsymbol{\nabla})$ and effective sheet carrier concentration (०) of samples implanted with different doses and annealed at $0.8 \mathrm{~J} / \mathrm{cm}^{2}$ and substrate (dotted line)

This work was made possible thanks to the FPI program (Grant No. BES-2005-7063) of the Ministerio de Educación y Ciencia, and was partially supported by the Projects NUMANCIA (No. S-0505/ENE/000310) founded by the Comunidad de Madrid and GENESIS-FV (No. CSD200600004) funded by the Spanish Consolider National Program and by U.C.M.-C.A.M. under Grant CCG07-UCM/TIC-2804.

\section{REFERENCES}

[1] A. Martí and A. Luque, "Next generation photovoltaics: high efficiency through full spectrum utilization," Institute of Physics Publishing, Bristol, UK, 2004.

[2] Luque and A. Martí, "Increasing the efficiency of ideal solar cells by photon induced transitions at intermediate levels," Phys. Rev. Lett., vol. 78 , no. 26 , pp. 5014-5017, Jun. 1997.

[3] M. A. Green, "Third generation of photovoltaics" (Springer, Berlinm Germany, 2005).

[4] W. Shockley and H. J. Queisser, "Detailed balance limit of efficiency of p-n junction solar cells," J. Appl. Phys., vol. 32, pp. 510-519, 1961.

[5] A. Luque et al., "Experimental analysis of the quasi-Fermi level split in quantum dot intermediate-band solar cells," Appl. Phys. Lett., vol. 87, no. 8, pp. 083505.1-083505.3, Aug. 2005.

[6] K. M. Yu, M. A. Scarpulla, R. Farshchi, O. D. Dubon and W. Walukiewicz, "Synthesis of highly mismatched alloys using ion implantation and pulsed laser annealing," Nucl. Instrum. Methods B, vol. 261, p. 1150, Mar. (2007).

[7] N. F. Mott, "Metal Insulator Transition," Rev. Mod. Phys., vol. 40, no. 4, pp. $677-683$, Oct. 1968

[8] S. Hocine and D. Mathiot, "Titanium diffusion in silicon," Appl. Phys. Lett., vol. 53, no. 14, pp. 1269-1271, Oct. 1988.

[9] K. M. Yu et al., "Multiband GaNAsP quaternary alloys," Appl. Phys. Lett., vol. 88, no. 9, pp. 092110.1-092110.3, Mar. 2006.

[10] P. Wahnón, Universidad Politécnica de Madrid, Madrid, Spain, private communication, Jul. 2008.

[11] J. Olea, M. Toledano-Luque, D. Pastor, G. González-Díaz and I. Mártil, "Titanium doped silicon layers with very high concentration," J. Appl. Phys., vol. 104, pp. 016105.1-3, Jul. 2008.

[12] M. H. Clark and K. S. Jones, "Strain compensation in boron-indium coimplanted laser thermal processed silicon," J. Appl. Phys., vol. 97, pp. 093525.1-4, Apr. 2005.

[13] D. B. Williams and B. C. Carter, "Transmission electron microscopy. II, Diffraction," Plenum Press, cop., New York, USA, 1996. 